\title{
Recent advances in hyaluronic acid based therapy for osteoarthritis
}

\author{
Steven Bowman ${ }^{1+}$, Mohamed E. Awad ${ }^{2 \dagger}$, Mark W. Hamrick ${ }^{3,4}$, Monte Hunter ${ }^{1}$ and Sadanand Fulzele ${ }^{1,4^{*}}$
}

\begin{abstract}
Osteoarthritis is a debilitating disease that has increased in prevalence across the world due to the aging population. Currently, physicians use a plethora of treatment strategies to try and slow down the progression of the disease, but none have been shown to ubiquitously treat and cure the disease. One of the strategies uses the high molecular weight molecule hyaluronic acid as either an injectable or oral supplement for treatment. Hyaluronic acid (HA) is a relatively new treatment that has shown varied results through several clinical trials. It can be used as a scaffold for engineering new treatments and several new preparations have just been added to the market. A comprehensive search was conducted through several search databases according our inclusion and exclusion criteria. This review included 44 prospective clinical trial investigating the feasibility and efficacy of HA injection for knee, hip, and ankle osteoarthritis. This review will take a closer look at hyaluronic acid and its properties, as well clinical effectiveness and future options.
\end{abstract}

Keywords: Osteoarthritis, Hyaluronic acid (HA), Treatment, Tissue engineering

\section{Introduction}

Osteoarthritis (OA) is a degenerative joint disease that frequently affects the hands and weight bearing joints of the body [1]. In United States, 52.5 million adults have been diagnosed with osteoarthritis according to data analyzed between 2010 and 2012 in the National Health Interview Survey (NHIS) [2]. In addition, OA is considered as one of the main causes of functional disability in (estimated) 22.7 million US adults [3]. The patient with OA is suffering not only from the persistent pain, stiffness and limited mobility. However, it also directly affects their quality of life with physical and/or mental co-morbidity [4]. OA substantially increases health care expenditures which is estimated around $\$ 128$ billion [5]. When considering productivity loss due to OA, estimates are between 0.25 and $0.50 \%$ of the Gross Domestic Product (GDP) [5].

Osteoarthritis (OA) is poorly understood because of its vast complexity and interplay of various biological

\footnotetext{
*Correspondence: sfulzele@augusta.edu

† Steven Bowman and Mohamed E. Awad contributed equally to this work

${ }^{1}$ Department of Orthopedics, Augusta University, 30904 Augusta, Georgia

Full list of author information is available at the end of the article
}

factors such as: genetic alterations, sex hormone deficit, and aging [6]. Many recent evidence has focused on molecular markers that implicated in the stress-induced senescent state of chondrocytes [7]. The term "Chondrosenescence" has been currently used to describe the age-dependent deterioration of chondrocyte function [8]. The therapeutic approaches for OA are limited because of its complex pathophysiology. According to the Osteoarthritis Research Society International (OARSI) Guidelines and recommendations for OA management, a core set of evidence based-modalities of therapy has been established [9]. These modalities included non-pharmacological such as patient education and awareness, physical exercise and rehabilitation aids. The pharmacological modalities vary from prescription of acetaminophen, non-selective NSAIDs (Nonsteroidal anti-inflammatory drugs) and selective COX-2 inhibitors agents and even opioid prescription. NSAIDs are the most prescribed agents for OA [10]. Despite NSAIDs established effectiveness in relieving the pain with OA its long term use is associated with potential harmful adverse effects. In addition, there is a wide heterogeneity in their personalized response because of the pharmacogenomics interactions [11]. The other potential non-operative therapeutic 
methods are chondroitin sulfate, glucosamine, and intraarticular injections of visco-supplements, corticosteroids, or blood-derived products [9]. However, there is a controversy about their complete efficacy and long-term safety in improving the patients symptomatically [12]. Remarkably, physical therapy such as mind-body exercise, strength training exercises and aerobic exercises has all shown some promising results in improving the OA prognosis as long as the patients are consistently compliant with their physical therapy regimen [13-15]. The nutritional supplements such as dimethyl sulphoxide (DMSO) and methylsulfonylmethane (MSM, present in green plants, fruits and vegetables) have been tried with limited success [16].

Over the last decades, there is an ongoing trend to use Intra-articular injections of either corticosteroids, analgesics/anti-inflammatory drugs, polymerized collagen, anti-cytokine drugs, or hyaluronic acid as alternative modalities to maximize the topical effect and minimize the systemic adverse effects [17]. Each injection has been shown to lower the pain in patients in some form, though hyaluronic acid treatments seem to be the safest and last the longest [17]. This review article highlights the current advances of Hyaluronic acid based therapy for osteoarthritic patients.

The literature search was performed through several search databases such as PubMed, Ovid via Medline, and Web of science using wide-spectrum keywords: Hyaluronic acid, hyaluronate injection, visco-supplements; intraarticular; knee, ankle, hip; osteoarthritis, cartilage degeneration. All prospective randomized controlled and retrospective observational cohort trials investigating the efficacy and safety of intra-articular injection of HA were considered for inclusion (Fig. 1).

\section{Hyaluronic acid physiology in synovial fluid of joint} Hyaluronate is a high molecular weight, ubiquitous molecule that naturally occurs within the cartilage and synovial fluid. It is composed of alternating $N$-acetyl$\mathrm{D}$-glucosamine and D-glucuronic acid residues attached by $\beta(1-4)$ and $\beta(1-3)$ bonds with molecular mass ranging from 6500 to $10,900 \mathrm{kDa}$ [18]. Its rheological characteristics involved in the main function of synovial fluid to serve as a lubricant, scavenger for free radicals, and for the regulation of cellular activities such as binding of proteins [19]. Its functions in the joint include lubrication, serving as a space filler to allow the joint to stay open, and the regulation of cellular activities such as binding of proteins [19]. During the progression of OA, the endogenous HA in the joint is depolymerized from being of a high molecular weight (6500-10,900 kDa) into a lower molecular weight (2700-4500 kDa), which consequently diminishes the mechanical and viscoelastic properties

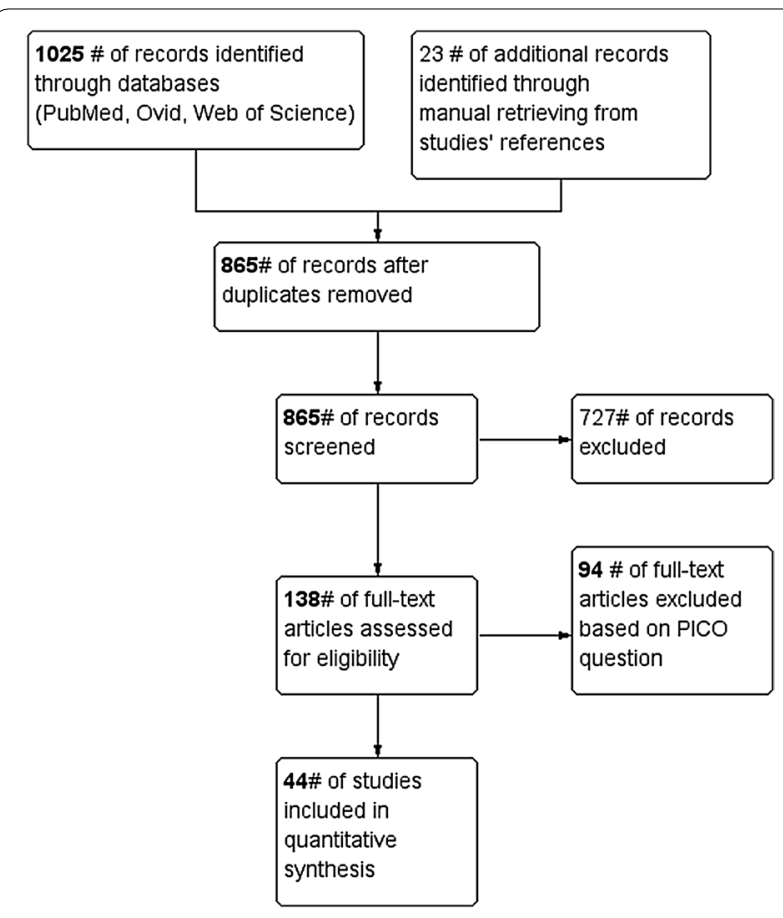

Fig. 1 Flow chart showing study identification, inclusion, and exclusion

of the synovial fluid in the affected joint $[18,20]$. Thus, exogenous HA injections have been clinically used to mitigate the macerated functions of the depolymerized endogenous HA of OA patients [20]. Although the exogenous HA does not restore and replace the full properties and activities of the depolymerized endogenous HA of the synovial fluid but it may induce satisfactory pain relief via several mechanisms [20]. These mechanisms include synthesis of proteoglycan and/or glycosaminoglycan, anti-inflammatory effect, and viscoelasticity maintenance [20]. Nevertheless, there is a clear heterogeneity in the Therapeutic trajectory for OA patients following HA injections. As some studies reporting an overall beneficial effect while others report that there is only a small benefit [21].

One of the potential reasons for the variable effect of HA treatments on OA patients is levels of hyaluronidases in a patient's synovial fluid. Hyaluronidases are a family of enzymes that degrade hyaluronic acid through cleaving the $\beta(1-4)$ linkages of HA, fracturing the large molecule into smaller pieces before degrading it [22].

\section{Hyaluronic acid and its preparations for treatment of $O A$}

HA is being administrated into OA patients via two main ways either oral administration or local injection [23, 24]. Several preparations of injectable HA used for clinical 
use include Synvisc ${ }^{\circledR}$ and Synvisc-One ${ }^{\circledR}$ (Genzyme); Gel-One ${ }^{\circledR}$ (Zimmer); Hyalgan ${ }^{\circledR}$ (Fidia); Supartz FX $^{\mathrm{TM}}$ (Bioventus); Orthovisc ${ }^{\circledR}$ (Anika); Euflexxa ${ }^{\circledR}$, previously named Nuflexxa (Savient); Monovisc ${ }^{\circledR}$ (Anika Therapeutics); and Gel-Syn ${ }^{\mathrm{TM}}$ (Institut Biochimique SA) [25]. Each product differs in many characteristics, including source (animal versus bacterial bio-fermentation using modified organisms), mean molecular weight ranging from (500 to $6000 \mathrm{kDa}$ ), distribution of molecular weight, molecular structure (linear, cross-linked or both), method of crosslinking, concentration $(0.8-30 \mathrm{mg} / \mathrm{mL})$, volume of injection (0.5-6.0 mL), and posology [26]. Although Animal source of HA (rooster combs) was considered as a traditional source for many years. However, many investigations have been performed to develop alternatives for obtaining HA, such as bio-fermentation using genetically modified organisms. This modified bacterial source is currently used as the main source as it's associated with lower costs and less side effects $[27,28]$.

Table 1 includes the characteristics of most common injectable hyaluronic acid products that are already approved by Food and Drug administration (FDA).

For oral HA treatment, the body absorbs the high molecular weight polymer as a decomposed 2-6 membered polysaccharide [29]. One proposed mechanism of action shows that ingested HA binds to Toll-like receptor- 4 and promotes the expressions of interleukin-10 and cytokine signaling, which both lead to anti-inflammation of arthritis [30]. a systematic review of 13 reports on oral HA clinical trials, Oe et al. found that patients that were on a highly pure HA regiment reported a beneficial effect on knee pain compared to placebo [23]. In terms of safety, it has been shown that on a 12 month study of 30 patients taking an oral HA regiment, no statistically significant negative side effects were seen [31].

As stated before, locally injected HA differs in many different characteristics. The most fundamental change is the molecular weight of the HA in the injection, and it was shown that there is no significant difference in the long term outcome regardless the preparation [32]. Unlike oral treatment, the complete HA molecule is introduced to the synovial fluid of the affected joint, providing a variety of different mechanisms for symptom relief [33]. These include enhancing the synthesis of extracellular matrix proteins, altering inflammatory mediators in order to shift away from degradation, reducing the motility of lymphocytes, and maintaining cartilage thickness, area and surface smoothness [24]. However, it must be stated that these are not the only proposed mechanism of actions for locally injected HA and further research trials needs to be performed in order to fully investigate the physiological effects of the treatment. Based on the study of 76 trials by Bellamy et al. locally injected HA treatment is an effective treatment for OA based on its effects of patients pain, function and patient global assessment [34]. In terms of safety, it has also been shown that there is no statistically significant negative side effects in patients receiving injection treatment [35].

Studies have shown that both local injections and oral supplementation of HA can combat OA symptoms, especially with those with early osteoarthritis [36]. Interestingly, Panuccio et al. showed that if these two types of treatments are combined, the oral supplementation of HA can extend the benefits of the injection treatments [37]. Thus, patients would not have to visit hospitals and receive the sometimes-uncomfortable injections as often [37]. Further randomized clinical trials are required to be designed in order to determine the exact outcomes of combined treatment.

\section{Hyaluronic acid based tissue engineering (modified therapy, biomaterial, scaffold and stem cells)}

Hyaluronic acid serves as a valuable material to create hydrogels that assist in healing because of its non-immunogenic properties, controlled biodegradability, biocompatible polymerization chemistry and multiple different reaction sites [38]. However, native HA is not useful and must be first cross-linked in order to provide stability and improve functionality of the gels [39]. In order to crosslink HA, different methods such as water-soluble carbodiimide crosslinking, polyvalent hydrazide crosslinking, divinyl sulfone crosslinking, disulfide crosslinking, and photo-crosslinking through glycidyl methacrylate-HA conjugation have been used [40].

Cross-linked HA hydrogels have several applications in the field of bioengineering. These include processes such as cell delivery, molecule delivery, cartilage tissue engineering, and development of micro-device systems [41]. Hydrogel scaffolds can be embedded with mesenchymal stem cells (MSCs) in order to boost the efficacy of regenerative capacity of MSCs [23, 25]. When paired with an HA hydrogel, MSCs have been shown to undergo chondrogenic differentiation, which leads to neo-cartilage formation and recovery of some of the degraded cartilage of patients with OA [26]. However, these MSCs also undergo hypertrophic phenotype changes as an adverse effect of being within the hydrogel scaffold, which lead to extensive calcification of the neo-cartilage matrix [27]. To combat the calcification and hypertrophic changes thus leading towards more chondrogenesis, specific HA hydrogel scaffolds are being engineered that facilitate the latter process and hinder the former processes [27, 28].

The most common molecule paired with HA scaffolds are growth factors, which recently have been shown to be able to recruit endogenous stem cells to a defect site and 


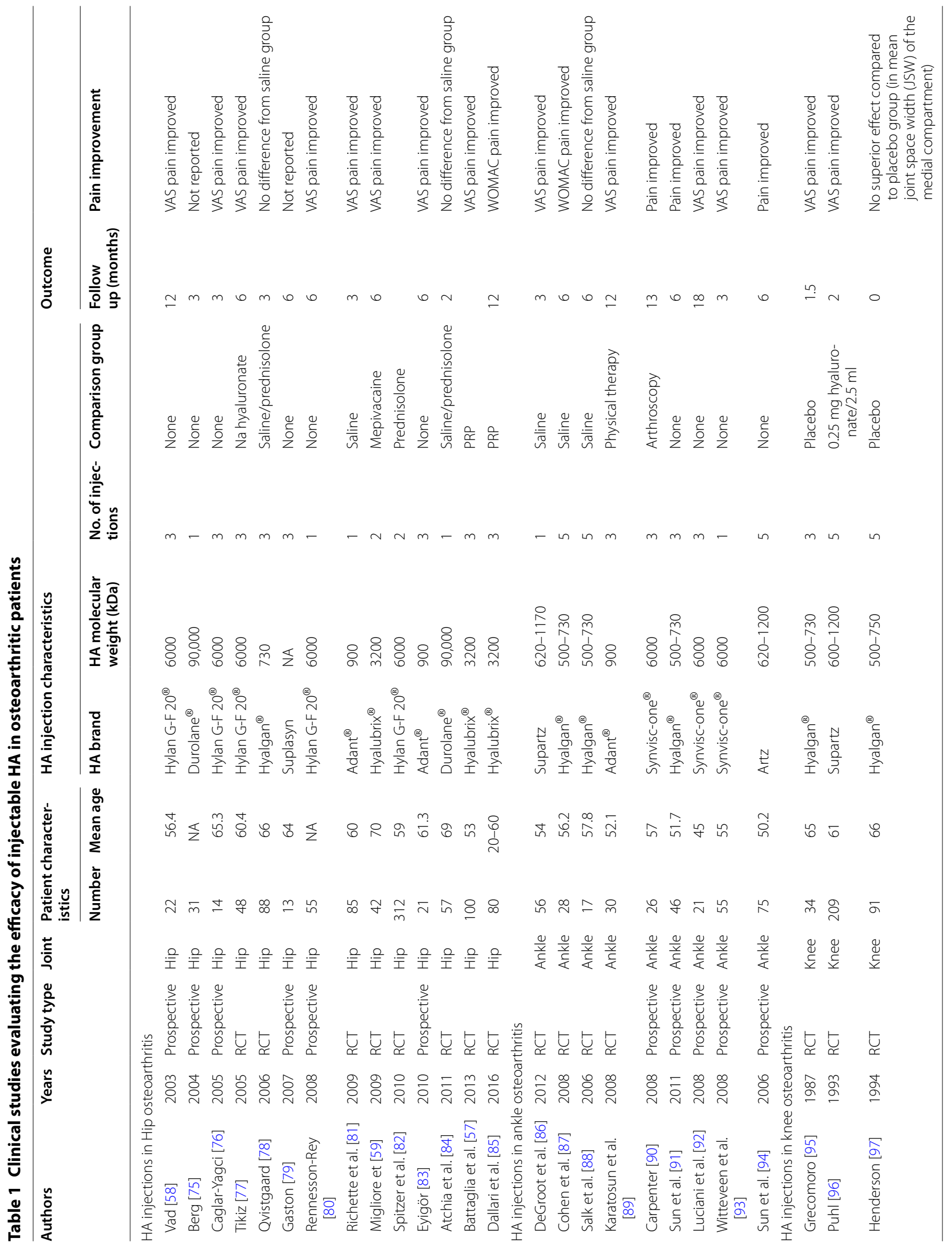




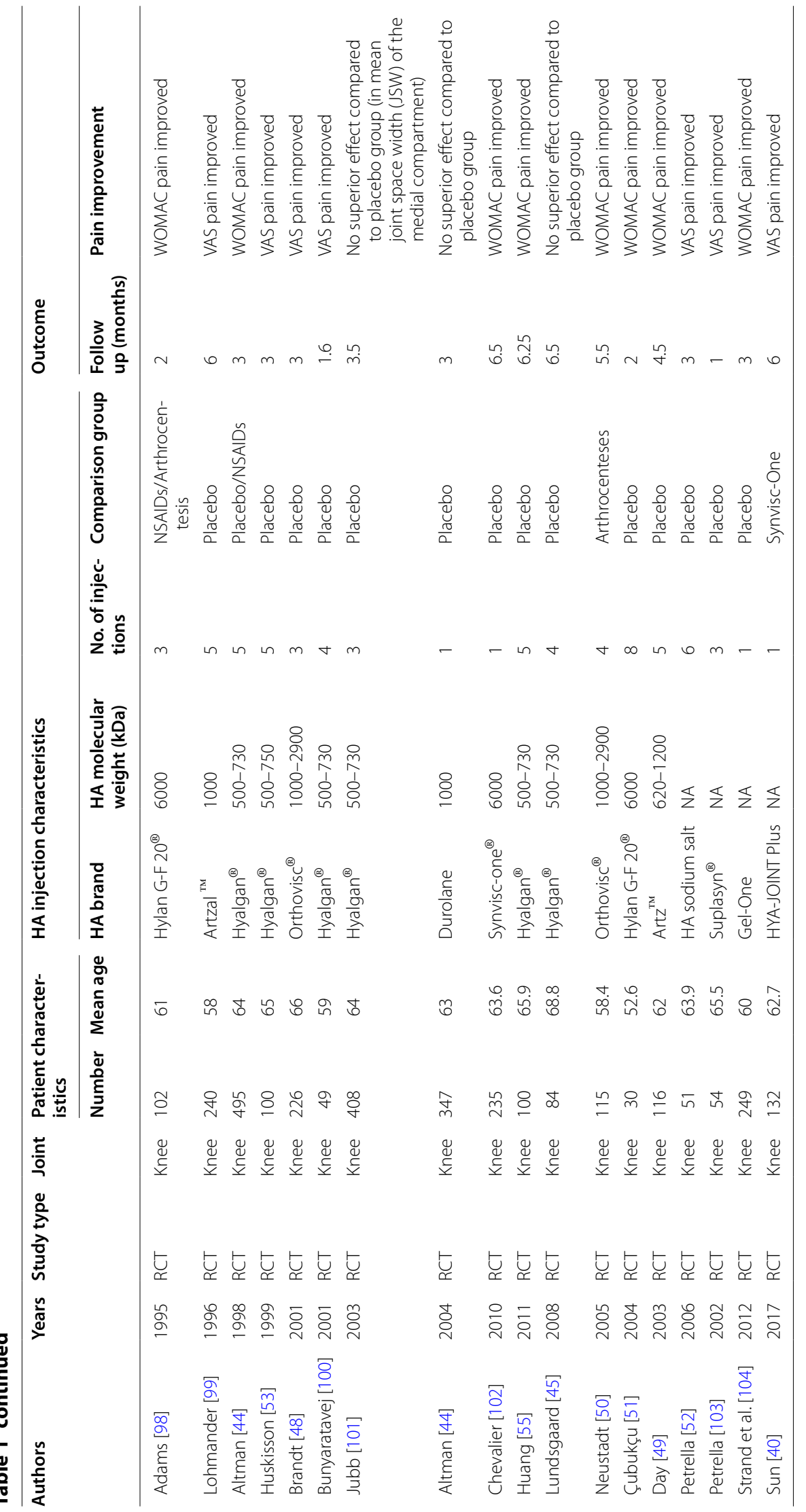


allow for de novo tissue regeneration [41]. With cartilage tissue engineering, chondrocytes can be encapsulated into hydrogel networks in order to treat the damaged cartilage tissue [41]. Taking advantage of the spatial control of certain types of HA hydrogels, microdevice systems are being developed that can encapsulate viable embryonic stem cells and then retrieved later using mechanical disruption [41]. These stem cells could then be used for treatment of diseases such as OA.

HA hydrogels are sometimes synthesized into scaffolds that can aid in the creation of new tissue [42]. In general, the scaffolds' modes of action are dependent on their physical properties, mass transport properties, and biological properties [42]. Specifically for HA, those stated properties are dependent on things such as the molecular weight of HA, whether HA is composited with another polymer, degree of grafting, crosslinker type and crosslink densities, as well as interaction with cell surface receptors [42]. With all these mechanisms in mind, it's easy to see how valuable HA is for creating scaffolds because of its natural properties that can be modified in a variety of different ways.

\section{Human clinical studies involving hyaluronic acid}

Improved hydrogels are continuously being made that seek to maximize the effect of treatment. One such is called Gel-One, which is composed of a product called Gel-200, a cross-linked hyaluronate hydrogel [43]. This product was first shown to produce chondroprotective, anti-inflammatory effects and long-lasting analgesia in OA mouse models [43]. Another new product, HYAJOINT Plus, was shown to produce a longer lasting and stronger effect on pain than compared to Synvisc-one, which is currently used by many physicians in intraarticular injections [44]. Furthermore, the product Cingal combined HA hydrogels with triamcinolone hexacetonide, a long acting corticosteroid previously shown to help with arthritis [45]. A clinical trial showed that Cingal provided immediate and long-term relief of osteoarthritis-related pain, stiffness, and function through 26 weeks when compared to saline [45]. Additionally, a new product named Cartistem paired HA hydrogels with human umbilical cord blood-derived mesenchymal stem cells has been used [46]. Unlike the usual autologous MSCs, these allogenic stem cells are isolated in a noninvasive manner and have shown a high expansion capacity, allowing a plethora of cells for therapeutic applications [46]. Moreover, it was previously shown in a sheep model of osteoarthritis that allogenic MSCs have a similar efficacy of treatment as autologous MSCs [34]. In a clinical trial, the product showed maturing repair tissue at 12 weeks and pain lowered at 24 weeks, both of which remained stable over 7 years of follow-up [33].
Over the last decades, several clinical trials have been developing many HA preparations and investigating their efficacy and safety. Although many studies have demonstrated that the use of intra-articular HA injection would be beneficial, non-surgical option for OA and may delay the need for joint replacement [47]. However, there is an ongoing controversy over the clinical effectiveness and sustainability of intra-articular injection of HA for OA patients. In 2004, Altman et al. [48] concluded that Durolane $^{\circledR}$ (a non-animal stabilized product with very high molecular weight $\mathrm{HA} ; 100,000 \mathrm{kDa}$ ) [49] is not beneficial and had no superiority over the placebo treated groups in terms of The Western Ontario and McMaster Universities Osteoarthritis Index (WOMAC) score and other efficacy parameters. In addition, Lundsgaard et al. [50] demonstrated that there is not any significant difference between the intra-articular injection of HA (Hylagan $\left.{ }^{\circledR} ; 6000 \mathrm{kDa}\right)$ and injection of physiological saline in patient with knee OA.

The influence of HA molecular mass on the clinical and functional efficacy remains debatable. In 2005, Karatosun and his colleagues [51] reported that there is no statistically significant difference between intra-articular injections of both high and low molecular weight hyaluronic acid for late-stage knee OA patients. Both groups experienced a substantial improvement in the outcome parameters at the latest follow-up. On the other hand, Berenbaum et al. [1] demonstrated that 3 weekly injections of GO-ON (intermediate MW HA; 800-1500 kDa) had statistical superiority ( $95 \% \mathrm{CI}$ all $>0, \mathrm{p}=0.021$ ) over Hyalgan $^{\circledR}$ (Low MW HA; 500-730 kDa) for knee OA symptoms over 6 months.

Many recent trials and meta-analyses have evaluated and proved the substantial superiority of injectable HA over the placebo group for pain relief and functional efficacy [52]. The clinical trial performed by Brandt et al. [48] and Day et al. [49] indicated that HA is safe and well-tolerated to induce a clinically significant improvement for patients with mild-to moderate knee OA. Furthermore, Neustadt et al. [53] reported that HMW-HA $\left(\right.$ Orthovisc $^{\circledR}$ ) also improve clinical outcome in advanced stage of OA (K-L grade 4).

One of the main controversial issues in this field is the timing and duration of injection and whether it may have an impact on its efficacy and sustainability. Cubukuc et al. [51] compared the intra-articular 3 weekly injections of Hylan G-F 20 and saline in OA patients. They reported that the optimal pain relief was noticed in HA group as early as 3rd week while functional improvement was seen at 8th week. In 2006, Patrella et al. [54] designed randomized controlled trial to determine the difference between three versus 6 consecutive weekly HA injections. They demonstrated that there are no 
differences between 3 and 6 HA injections in regard of pain, function, and patient satisfaction. In 1999, Huskisson et al. [53] demonstrated that 5 weekly intra-articular injections of sodium hyaluronate (Hyalgan A) would provide a symptomatic improvement which persisted for 6 months. Recently, two large, controlled randomized clinical trial confirms that 5 weekly IA injections of HA (Hyalgan) in patients with knee OA provided sustained relief of pain and improved patient function, and were at least as effective with fewer adverse reactions as continuous treatment with naproxen for 26 weeks $[55,56]$. From the cost-effectiveness stem point, Hyalgan ${ }^{\circledR}$ and Supartz ${ }^{\circledR}$ are considered as economically feasible to provide a rapid pain relief and functional outcomes when compared to Orthovisc ${ }^{\circledR}$ and Synvisc ${ }^{\circledR}$. Despite the more number of injections required in Hyalgan ${ }^{\circledR}$ and Supartz ${ }^{\circledR}$ courses, it still costs healthcare plans less than Synvisc ${ }^{\circledR}$ [57].

Many trials have been performed to investigate the role of intra-articular injection of HA in alleviating the symptoms in hip osteoarthritis. A randomized clinical trial compared HA injection to platelet rich plasma (PRP) injection, Battaglia et al. [58] demonstrated that HA is superior to PRP in patients with symptomatic hip $\mathrm{OA}$ in term of pain relief and functional improvement. In 2003, Vad et al. [59] reported that three HA injections would be safe and an essential option for mild-to moderate hip OA to produce a rapid pain relief. However, they showed no efficacy for the patients with severe hip OA. Apart from that Intra-articular injection of low and high molecular weight HA was notably effective in relieving the pain, it was also associated with reduction of $48.2 \%$ of NSAIDs consumption at the 3rd month when compared with baseline values [60]. The conclusion of meta-analysis of 26 clinical trials indicated that HA injection would be consider as the best conservative line for hip OA with substantial pain relief and function amelioration. However, there is no clear evidence to prove its ability to modify the morphological or radiological changes of the pathological hip [61].

A recent meta-analysis including nine clinical trials exploring the effectiveness of intra-articular HA injection for the treatment of ankle OA and to investigate the effects of modified regimens of HA. Its results suggest that intra-articular HA administration can significantly reduce pain for patients with ankle OA. In addition, Intra-articular HA was likely superior to other conservative therapy. They suggested the use of multiple doses with an appropriate injection volume would achieve maximum therapeutic effects [62].

In 2013, a pilot study examined the effectiveness of the intra-articular injection of Euflexxa in 22 patients with osteoarthritis of the subtalar joint [63]. Euflexxa is a bioengineered $1 \%$ sodium $\mathrm{HA}$ that does not require cross-linking, has a molecular weight range of 2.4$3.6 \mathrm{mDa}$ and is synthesized via controlled fermentation [55]. The study showed that patients significantly experienced an improvement in American Orthopedic Foot and Ankle Society Ankle Hind foot scores and visual analog scale assessment as well as longer tolerated walking distance [63]. Likewise, a clinical trial was conducted to explore the effectiveness and safety of the HA-chondroitin sulfate medication Arthrum HCS [64]. Arthrum HCS is a mixture of $40 \mathrm{mg}$ hyaluronic acid and $40 \mathrm{mg}$ chondroitin sulfate in a 2-mL solution [64]. They investigated one hundred and twelve patients and showed that Arthrum lowered the WOMAC sub score A in assessments at 1, 3 and 6-month intervals after treatment [64]. Additionally, the trial showed that patients had improved mobility and reduced their consumption of analgesics [64]. In addition, Xin et al. designed a clinical study to compare the efficacy and safety of two different sodium hyaluronate drugs, Adant ${ }^{\circledR}$ and Artz ${ }^{\circledR}$ [65]. The main difference between the two drugs is the manufacturing process, with Adant ${ }^{\circledR}$ being manufactured by fermentation and Artz ${ }^{\circledR}$ being manufactured by extraction of cockscomb [65]. The study concluded that both drugs showed a significant reduction in VAS scores while not showing a significant difference in efficacy and safety [65]. Moreover, another clinical trial was conducted to see the safety and efficacy of Hyalubrix via an observational study of normal medical practice [66]. Hyalubrix is a sterile, nonpyrogenic solution of HA sodium salt with a molecularweight around $1500 \mathrm{kDa}$ that is produced by bacterial fermentation [67]. The study showed that there was a statistically significant improvement in VAS, HAQ and EuroQol scores while only showing a $0.8 \%$ adverse event rate [66].

\section{Animal studies and future directions for the clinical use of hyaluronic acid}

As with all novel disease treatments, animal studies are continuously being designed in order to determine the safety and efficacy of treatment. Some examples for osteoarthritis animal studies include a novel treatment that combines HA, chondroitin sulfate and glucosamine (HACSNAG) [68]. Sükür et al. used a rat model with early stage OA and showed that this novel compound (HACSNAG) provided a more chondroprotective effect in the rats' cartilage when compared to HA treatment alone [68]. In 2016, Tamura et al. examined a novel conjugate composed of HA and methotrexate called DK226 [69]. Methotrexate has been one of the most widely used medications for rheumatoid arthritis and has recently been shown to help in osteoarthritis of the knee [69]. Their results showed that the intra-articular injection of DK226 showed similar anti-arthritic effects as oral methotrexate 
but eliminated the harmful side-effects that usually come with oral treatment [69].

A study performed by Ishikawa et al. to investigate the biocompatibility and adverse effects of the aforementioned medication Gel-200 [70]. They used subcutaneous air pouch rat model as well as knee joints of normal rabbits [70]. They concluded with showing that Gel-200 did not induce any granulomatous inflammation nor a significant thickening of the fibrous belt often seen in the air pouch models [70]. This was compared to the hylan G-F 20 medication Synvisc which did the exact opposite and showed a granulomatous inflammation and thickening of the fibrous belt [70].

Additionally, nanoparticles composed of poly (D,Llactic acid) (PLA) or poly(D,L-lactic-co-glycolic acid) (PLGA) covered by chemically esterified amphiphilic HA are being considered as drug carriers for treatment of OA [71]. A study using healthy rat models was designed to examine if the nanoparticles had a toxic effect on the model knees [71]. Zille et al. showed that the nanoparticles did not modify the synovial membranes and did not upregulate the cytokines IL- $1 \beta$ and TNF- $\alpha$ [71]. A separate study was done using $350 \mathrm{~g}$ female Sprague- Dawley rats to study if the nanoparticles were retained in the joint and concluded that in $70 \%$ of the rats, the nanoparticles were retained for at least a week [72].

\section{Strengths and limitations of hyaluronic acid treatment}

As stated before, HA treatment has shown many beneficial effects in studies and experiments such as intraarticular lubrication, anti-inflammatory, analgesic and chondroprotective effects, among others [20]. However, considering its cost it is not always the recommended treatment for OA patients. According to both the Osteoarthritis Research Society International 2012 guideline and the American College of Rheumatology 2013 guidelines, HA treatment is neither recommended or discouraged because of the inconsistency of clinical studies $[73,74]$. For a plethora of the studies both societies researched, a large placebo effect was seen which limited the scope of the data [73, 74]. The treatment also does not provide an immediate relief to most patients, as studies have shown that it takes about 5 weeks before patients feel the full effect of the treatment [75]. Despite the demonstrated efficacy and the safety of HA products, there are few associated side effects that mostly limited to local pain and swelling with frequent injections [76]. Further investigations are still required to obtain specially designated molecular mass HA to optimize the clinical effect and extend its applications. In addition, more randomized controlled trials with a large sample to test not only the efficacy of HA versus the other established therapies of OA, but also the different products, dosages and the optimal number of injections.

\section{Conclusion}

Osteoarthritis is a debilitating disease that affects a large portion of the population. As the general age of the population continues towards an older age, the prevalence of the disease is only going to go up. Therefore, more research is needed in order to fully control the disease and its side effects. Hyaluronic acid is a potential bright spot for helping lower the side effects. Its effectiveness is due to the many methods of actions it deploys, including lubrication, anti-inflammatory and chondroprotective effects. Treatment can be done both orally and through intra-articular injections. New products are continuously being developed that change the composition of the molecule as well as pairing it with other drugs to maximize the effect. Hyaluronic acid treatment shows a lot of potential that will hopefully be discovered through continued research.

\section{Authors' contributions}

$\mathrm{SB}, \mathrm{MEA}, \mathrm{MH}, \mathrm{MWH}$ and SF each made substantive intellectual contributions to the work described in this review paper. SF, SB and MH conceived the idea for the manuscript, and drafted the initial manuscript with $\mathrm{MHW}$. MH, and MEA provide clinical relevance and insight. SF, MH and MEA made the final edits to the manuscript. All authors read and approved the final manuscript.

\section{Author details \\ 1 Department of Orthopedics, Augusta University, 30904 Augusta, Georgia. \\ 2 Department of Oral Biology, Augusta University, Augusta, Georgia. ${ }^{3}$ Depart- ment of Cell Biology and Anatomy, Augusta University, Augusta, Georgia. \\ ${ }^{4}$ Institute of Regenerative and Reparative Medicine, Augusta University, Augusta, Georgia.}

\section{Acknowledgements}

We like to thank department of orthopaedic surgery for their support.

\section{Competing interests}

The authors declare that they have no competing interests.

\section{Availability of data and materials}

All of the data and material presented and discussed in this review article is available in the published articles listed below in the reference section.

\section{Funding}

This work is supported by Department of Orthopaedic Surgery, Augusta, GA.

Ethics approval and consent to participate

Not applicable.

\section{Publisher's Note}

Springer Nature remains neutral with regard to jurisdictional claims in published maps and institutional affiliations.

Received: 1 November 2017 Accepted: 22 December 2017

Published online: 16 February 2018

\footnotetext{
References

1. Berenbaum F (2013) Osteoarthritis as an inflammatory disease (osteoarthritis is not osteoarthrosis!). Osteoarthr Cartil 21(1):16-21
} 
2. CDC (2013) Prevalence of doctor-diagnosed arthritis and arthritisattributable activity limitation-United States, 2010-2012. MMWR 62:869-873

3. Barbour K et al (2014) Meeting physical activity guidelines and the risk of incident knee osteoarthritis: the Johnston County Osteoarthritis Project. Arthritis Care Res (Hoboken) 66(1):139-146

4. Moskowitz RW (2009) The burden of osteoarthritis: clinical and qualityof-life issues. Am J Manag Care 15(8 Suppl):S223-S229

5. Puig-Junoy J, Ruiz Zamora A (2015) Socio-economic costs of osteoarthritis: a systematic review of cost-of-illness studies. Semin Arthritis Rheum 44(5):531-541

6. Herrero-Beaumont $\mathrm{G}$ et al (2017) Clinical settings in knee osteoarthritis: pathophysiology guides treatment. Maturitas 96:54-57

7. Zhang W et al (2008) OARSI recommendations for the management of hip and knee osteoarthritis, Part II: OARSI evidence-based, expert consensus guidelines. Osteoarthritis Cartilage 16(2):137-162

8. Osteoarthritis Fact Sheet (2017) https://www.cdc.gov/arthritis/basics/ osteoarthritis.htm. Accessed 24 May 2017

9. Brosseau L et al (2017) The Ottawa panel clinical practice guidelines for the management of knee osteoarthritis. Part one: introduction, and mind-body exercise programs. Clin Rehabil 31(5):582-595

10. Brosseau $L$ et al (2017) The Ottawa panel clinical practice guidelines for the management of knee osteoarthritis. Part two: strengthening exercise programs. Clin Rehabil 31(5):596-611

11. Brosseau $L$ et al (2017) The Ottawa panel clinical practice guidelines for the management of knee osteoarthritis. Part three: aerobic exercise programs. Clin Rehabil 31(5):612-624

12. Brien $S$, Prescott $P$, Lewith $G$ (2011) Meta-analysis of the related nutritional supplements dimethyl sulfoxide and methylsulfonylmethane in the treatment of osteoarthritis of the knee. Evid Based Complement Alternat Med 2011:528403

13. Crofford $L J$ (2013) Use of NSAIDs in treating patients with arthritis. Arthritis Res Ther 15(Suppl 3):S2

14. da Costa BR et al (2016) Effectiveness of non-steroidal anti-inflammatory drugs for the treatment of pain in knee and hip osteoarthritis: a network meta-analysis. Lancet 387(10033):2093-2105

15. lannitti T, Lodi D, Palmieri B (2011) Intra-articular injections for the treatment of osteoarthritis: focus on the clinical use of hyaluronic acid. Drugs R\&D 11(1):13-27

16. Balazs EA et al (1967) Hyaluronic acid in synovial fluid. I. Molecular parameters of hyaluronic acid in normal and arthritis human fluids. Arthritis Rheum 10(4):357-376

17. Laurent TC, Laurent UB, Fraser JR (1996) The structure and function of hyaluronan: an overview. Immunol Cell Biol 74(2):A1-A7

18. Altman RD et al (2015) The mechanism of action for hyaluronic acid treatment in the osteoarthritic knee: a systematic review. BMC Musculoskelet Disord 16:321

19. Ayhan E, Kesmezacar H, Akgun I (2014) Intraarticular injections (corticosteroid, hyaluronic acid, platelet rich plasma) for the knee osteoarthritis. World J Orthop 5(3):351-361

20. Stern R, Jedrzejas MJ (2006) Hyaluronidases: their genomics, structures, and mechanisms of action. Chem Rev 106(3):818-839

21. Oe M et al (2015) Oral hyaluronan relieves knee pain: a review. Nutr J 15:11

22. Moreland LW (2003) Intra-articular hyaluronan (hyaluronic acid) and hylans for the treatment of osteoarthritis: mechanisms of action. Arthritis Res Ther 5(2):54-67

23. Bannuru RR et al (2011) Therapeutic trajectory following intra-articular hyaluronic acid injection in knee osteoarthritis - meta-analysis. Osteoarthritis Cartilage 19(6):611-619

24. Cooper $C$, Rannou F, Richette $P$, Bruyère $O$, Al-Daghri N, Altman RD, Brandi ML, Collaud Basset S, Herrero-Beaumont G, Migliore A, Pavelka K, Uebelhart D, Reginster JY (2017) Use of intraarticular hyaluronic acid in the management of knee osteoarthritis in clinical practice. Arthritis Care Res (Hoboken) 69(9):1287-1296

25. Hisada $\mathrm{N}$ et al (2008) Low-molecular-weight hyaluronan permeates through human intestinal Caco-2 cell monolayers via the paracellular pathway. Biosci Biotechnol Biochem 72(4):1111-1114

26. Asari A, Kanemitsu T, Kurihara H (2010) Oral administration of high molecular weight hyaluronan (900 kDa) controls immune system via Toll-like receptor 4 in the intestinal epithelium. J Biol Chem 285(32):24751-24758

27. Tashiro T et al (2012) Oral administration of polymer hyaluronic acid alleviates symptoms of knee osteoarthritis: a double-blind, placebocontrolled study over a 12-month period. Sci World J 2012:167928

28. Shewale AR, Barnes CL, Fischbach LA, Ounpraseuth ST, Painter JT, Martin BC (2017) Comparison of low-, moderate-, and high-molecularweight hyaluronic acid injections in delaying time to knee surgery. J Arthroplasty 32(10):2952-2957.e21

29. Balazs EA (2004) Viscosupplementation for treatment of osteoarthritis: from initial discovery to current status and results. Surg Technol Int 12:278-289

30. Bellamy $\mathrm{N}$ et al (2006) Viscosupplementation for the treatment of osteoarthritis of the knee. Cochrane Database Syst Rev 19(2):Cd005321

31. Nguyen C, Rannou F (2017) The safety of intra-articular injections for the treatment of knee osteoarthritis: a critical narrative review. Expert Opin Drug Saf 16(8):897-902

32. Ricci M et al (2017) Clinical comparison of oral administration and viscosupplementation of hyaluronic acid (HA) in early knee osteoarthritis. Musculoskelet Surg 101(1):45-49

33. Panuccio E, Memeo A, Richetta S (2015) Evaluation of the combined treatment of oral viscosupplementation with hyaluronic acid intra-articular injection on symptomatic knee osteoarthritis. Clin Ter 166(5):e321-e326

34. Baier Leach J et al (2003) Photocrosslinked hyaluronic acid hydrogels: natural, biodegradable tissue engineering scaffolds. Biotechnol Bioeng 82(5):578-589

35. Xu X et al (2012) Hyaluronic acid-based hydrogels: from a Natural Polysaccharide to Complex Networks. Soft Matter 8(12):3280-3294

36. Fakhari A (2012) Biomedical application of hyaluronic acid nanoparticles. PhD thesis

37. Burdick JA, Prestwich GD (2011) Hyaluronic acid hydrogels for biomedical applications. Adv Mater 23(12):H41-H56

38. Collins MN, Birkinshaw C (2013) Hyaluronic acid based scaffolds for tissue engineering-A review. Carbohyd Polym 92(2):1262-1279

39. Yoshioka Ket al (2014) Pharmacological effects of novel cross-linked hyaluronate, Gel-200, in experimental animal models of osteoarthritis and human cell lines. Osteoarthr Cartil 22(6):879-887

40. Sun SF et al (2017) Comparison of single intra-articular injection of novel hyaluronan (HYA-JOINT Plus) with synvisc-one for knee osteoarthritis: a Randomized, Controlled, Double-Blind Trial of Efficacy and Safety. J Bone Joint Surg Am 99(6):462-471

41. Hangody L et al. Intraarticular injection of a cross-linked sodium hyaluronate combined with triamcinolone hexacetonide (cingal) to provide symptomatic relief of osteoarthritis of the knee: A Randomized, Double-Blind, Placebo-Controlled Multicenter Clinical Trial. Cartilage, 2017: 1947603517703732

42. Park YB et al (2017) Cartilage regeneration in osteoarthritic patients by a composite of allogeneic umbilical cord blood-derived mesenchymal stem cells and hyaluronate hydrogel: results from a clinical trial for safety and proof-of-concept with 7 years of extended follow-Up. Stem Cells Transl Med 6(2):613-621

43. Waddell DD, Bricker DC (2007) Total knee replacement delayed with Hylan G-F 20 use in patients with grade IV osteoarthritis. J Manag Care Pharm 13(2):113-121

44. Altman RD et al (2004) Efficacy and safety of a single intra-articular injection of non-animal stabilized hyaluronic acid (NASHA) in patients with osteoarthritis of the knee. Osteoarthr Cartil 12(8):642-649

45. Lundsgaard C et al (2008) Intra-articular sodium hyaluronate $2 \mathrm{~mL}$ versus physiological saline $20 \mathrm{~mL}$ versus physiological saline $2 \mathrm{~mL}$ for painful knee osteoarthritis: a randomized clinical trial. Scand J Rheumatol 37(2):142-150

46. Karatosun $\mathrm{V}$ et al (2005) Comparison of two hyaluronan drugs in patients with advanced osteoarthritis of the knee. A prospective, randomized, double-blind study with long term follow-up. Clin Exp Rheumatol 23(2):213-218

47. Xing D, Wang B, Liu Q, Ke Y, Xu Y, Li Z, Lin J (2016) Intra-articular hyaluronic acid in treating knee osteoarthritis: a PRISMA-compliant systematic review of overlapping meta-analysis. Sci Rep 6:32790

48. Brandt KD et al (2001) Efficacy and safety of intraarticular sodium hyaluronate in knee osteoarthritis. ORTHOVISC Study Group. Clin Orthop Relat Res 385:130-143 
49. Day R et al (2004) A double blind, randomized, multicenter, parallel group study of the effectiveness and tolerance of intraarticular hyaluronan in osteoarthritis of the knee. J Rheumatol 31(4):775-782

50. Neustadt D et al (2005) Clinical effects of intraarticular injection of high molecular weight hyaluronan (Orthovisc) in osteoarthritis of the knee: a randomized, controlled, multicenter trial. J Rheumatol 32(10):1928-1936

51. Cubukcu D et al (2005) Hylan G-F 20 efficacy on articular cartilage quality in patients with knee osteoarthritis: clinical and MRI assessment. Clin Rheumatol 24(4):336-341

52. Petrella RJ, Petrella M (2006) A prospective, randomized, double-blind, placebo controlled study to evaluate the efficacy of intraarticular hyaluronic acid for osteoarthritis of the knee. J Rheumatol 33(5):951-956

53. Huskisson EC, Donnelly S (1999) Hyaluronic acid in the treatment of osteoarthritis of the knee. Rheumatology 38(7):602-607

54. Altman RD et al (2011) Safety and efficacy of retreatment with a bioengineered hyaluronate for painful osteoarthritis of the knee: results of the open-label Extension Study of the FLEXX Trial. Osteoarthr Cartil 19(10):1169-1175

55. Huang TL et al (2011) Intra-articular injections of sodium hyaluronate (Hyalgan $\left.\left({ }^{\circledR}\right)\right)$ in osteoarthritis of the knee. A randomized, controlled, double-blind, multicenter trial in the asian population. BMC Musculoskelet Disord 12:221

56. Dasa V, DeKoven M, Sun K, Scott A, Lim S (2016) Clinical and cost outcomes from different hyaluronic acid treatments in patients with knee osteoarthritis: evidence from a US health plan claims database. Drugs Context 5:212296

57. Battaglia M et al (2013) Efficacy of ultrasound-guided intra-articular injections of platelet-rich plasma versus hyaluronic acid for hip osteoarthritis. Orthopedics 36(12):e1501-e1508

58. Vad VB et al (2003) Role of hylan G-F 20 in treatment of osteoarthritis of the hip joint. Arch Phys Med Rehabil 84(8):1224-1226

59. Migliore A et al (2009) Comparative, double-blind, controlled study of intra-articular hyaluronic acid (Hyalubrix $\left({ }^{\circledR}\right)$ ) injections versus loca anesthetic in osteoarthritis of the hip. Arthritis Res Ther 11(6):R183

60. Piccirilli E et al (2016) Viscosupplementation with intra-articular hyaluronic acid for hip disorders. A systematic review and meta-analysis. Muscles Ligaments Tendons J 6(3):293-299

61. Chang KV et al (2013) Effectiveness of intra-articular hyaluronic acid for ankle osteoarthritis treatment: a systematic review and meta-analysis. Arch Phys Med Rehabil 94(5):951-960

62. Mei-Dan $\mathrm{O}$ et al (2013) Intra-articular injections of hyaluronic acid in osteoarthritis of the subtalar joint: a pilot study. J Foot Ankle Surg 52(2):172-176

63. Rivera F et al (2016) Effectiveness of intra-articular injections of sodium hyaluronate-chondroitin sulfate in knee osteoarthritis: a multicenter prospective study. J Orthop Traumatol 17(1):27-33

64. Xin Y et al (2016) The efficacy and safety of sodium hyaluronate injection (Adant $\left({ }^{\circledR}\right)$ ) in treating degenerative osteoarthritis: a multi-center, randomized, double-blind, positive-drug parallel-controlled and noninferiority clinical study. Int J Rheum Dis 19(3):271-278

65. Foti $C$ et al (2011) A prospective observational study of the clinical efficacy and safety of intra-articular sodium hyaluronate in synovial joints with osteoarthritis. Eur J Phys Rehabil Med 47(3):407-415

66. Migliore A et al (2009) Comparative, double-blind, controlled study of intra-articular hyaluronic acid (Hyalubrix $\left({ }^{\circledR}\right)$ ) injections versus local anesthetic in osteoarthritis of the hip. Arthritis Res Ther 11(6):R183-R183

67. Şükür E et al (2016) Comparison of the chondroprotective effect of a novel hydrogel compound and traditional hyaluronate on rat cartilage in a papain-induced osteoarthritis model. Acta Orthopaedica et Traumatologica Turcica 50(4):458-463

68. Tamura T et al (2016) Novel hyaluronic acid-methotrexate conjugate suppresses joint inflammation in the rat knee: efficacy and safety evaluation in two rat arthritis models. Arthritis Res Ther 18:79

69. Ishikawa M et al (2014) Biocompatibility of cross-linked hyaluronate (Gel-200) for the treatment of knee osteoarthritis. Osteoarthr Cartil 22(11):1902-1909

70. Zille H et al (2010) Evaluation of intra-articular delivery of hyaluronic acid functionalized biopolymeric nanoparticles in healthy rat knees. Biomed Mater Eng 20(3):235-242
71. Morgen M et al (2013) Nanoparticles for improved local retention after intra-articular injection into the knee joint. Pharm Res 30(1):257-268

72. McAlindon TE et al (2014) OARSI guidelines for the non-surgical management of knee osteoarthritis. Osteoarthritis Cartilage 22(3):363-388

73. Hochberg MC et al (2012) American College of Rheumatology 2012 recommendations for the use of nonpharmacologic and pharmacologic therapies in osteoarthritis of the hand, hip, and knee. Arthritis Care Res (Hoboken) 64(4):465-474

74. Gigis I et al (2016) Comparison of two different molecular weight intra-articular injections of hyaluronic acid for the treatment of knee osteoarthritis. Hippokratia 20(1):26-31

75. Berg P, Olsson U (2004) Intra-articular injection of non-animal stabilised hyaluronic acid (NASHA) for osteoarthritis of the hip: a pilot study. Clin Exp Rheumatol 22(3):300-306

76. Caglar-Yagci H et al (2005) Safety and efficacy of ultrasound-guided intra-articular hylan G-F 20 injection in osteoarthritis of the hip: a pilot study. Rheumatol Int 25(5):341-344

77. Tikiz C et al (2005) Comparison of the efficacy of lower and higher molecular weight viscosupplementation in the treatment of hip osteoarthritis. Clin Rheumatol 24(3):244-250

78. Qvistgaard E et al (2006) Intra-articular treatment of hip osteoarthritis: a randomized trial of hyaluronic acid, corticosteroid, and isotonic saline. Osteoarthritis Cartilage 14(2):163-170

79. Gaston MS, Tiemessen CH, Philips JE (2007) Intra-articular hip viscosupplementation with synthetic hyaluronic acid for osteoarthritis: efficacy, safety and relation to pre-injection radiographs. Arch Orthop Trauma Surg 127(10):899-903

80. Rennesson-Rey B, Rat AC, Chary-Valckenaere I, Bettembourg-Brault I, Juge N, Dintinger H, Pourel J, Loeuille D (2008) Does joint effusion influence the clinical response to a single Hylan GF-20 injection for hip osteoarthritis? Joint Bone Spine 75(2):182-188

81. Richette $P$ et al (2009) Effect of hyaluronic acid in symptomatic hip osteoarthritis: a multicenter, randomized, placebo-controlled trial. Arthritis Rheum 60(3):824-830

82. Spitzer Al, Bockow BI, Brander VA, Yates JW, MacCarter DK, Gudger GK, Haller S, Lake SL, Magilavy DB (2010) Hylan G-F 20 improves hip osteoarthritis: a prospective, randomized study. Phys Sportsmed 38(2):35-47

83. Eyigor C et al (2010) Efficacy of intraarticular hyaluronic acid injection through a lateral approach under fluoroscopic control for advanced hip osteoarthritis. Agri 22(4):139-144

84. Atchia l et al (2011) Efficacy of a single ultrasound-guided injection for the treatment of hip osteoarthritis. Ann Rheum Dis 70(1):110-116

85. Dallari D et al (2016) Ultrasound-guided injection of platelet-rich plasma and hyaluronic acid, separately and in combination, for hip osteoarthritis: a Randomized Controlled Study. Am J Sports Med 44(3):664-671

86. DeGroot $\mathrm{H}$ 3rd et al (2012) Intra-articular injection of hyaluronic acid is not superior to saline solution injection for ankle arthritis: a randomized, double-blind, placebo-controlled study. J Bone Joint Surg Am 94(1):2-8

87. Cohen MM et al (2008) Safety and efficacy of intra-articular sodium hyaluronate (Hyalgan) in a randomized, double-blind study for osteoarthritis of the ankle. Foot Ankle Int 29(7):657-663

88. Salk RS et al (2006) Sodium hyaluronate in the treatment of osteoarthritis of the ankle: a controlled, randomized, double-blind pilot study. J Bone Joint Surg Am 88(2):295-302

89. Karatosun $V$ et al (2008) Intra-articular hyaluronic acid compared to exercise therapy in osteoarthritis of the ankle. A prospective randomized trial with long-term follow-up. Clin Exp Rheumatol 26(2):288-294

90. Carpenter B, Motley $T$ (2008) The role of viscosupplementation in the ankle using hylan G-F 20. J Foot Ankle Surg 47(5):377-384

91. Sun SF et al (2006) Efficacy of intra-articular hyaluronic acid in patients with osteoarthritis of the ankle: a prospective study. Osteoarthr Cartil 14(9):867-874

92. Luciani D et al (2008) Viscosupplementation for grade II osteoarthritis of the ankle: a prospective study at 18 months' follow-up. Chir Organi Mov 92(3):155-160

93. Witteveen AG et al (2008) A prospective multi-centre, open study of the safety and efficacy of hylan G-F 20 (Synvisc) in patients with symptomatic ankle (talo-crural) osteoarthritis. Foot Ankle Surg 14(3):145-152 
94. Sun SF et al (2011) The effect of 3 weekly intra-articular injections of hyaluronate on pain, function, and balance in patients with unilateral ankle arthritis. J Bone Joint Surg Am 93(18):1720-1726

95. Grecomoro G, Martorana U, Di Marco C (1987) Intra-articular treatment with sodium hyaluronate in gonarthrosis: a controlled clinical trial versus placebo. Pharmatherapeutica 5(2):137-141

96. Puhl W et al (1993) Intra-articular sodium hyaluronate in osteoarthritis of the knee: a multicenter, double-blind study. Osteoarthr Cartil 1(4):233-241

97. Henderson EB et al (1994) Intra-articular injections of $750 \mathrm{kD}$ hyaluronan in the treatment of osteoarthritis: a randomised single centre double-blind placebo-controlled trial of 91 patients demonstrating lack of efficacy. Ann Rheum Dis 53(8):529-534

98. Adams ME et al (1995) The role of viscosupplementation with hylan G-F 20 (Synvisc) in the treatment of osteoarthritis of the knee: a Canadian multicenter trial comparing hylan G-F 20 alone, hylan G-F 20 with non-steroidal anti-inflammatory drugs (NSAIDs) and NSAIDs alone. Osteoarthr Cartil 3(4):213-225

99. Lohmander LS et al (1996) Intra-articular hyaluronan injections in the treatment of osteoarthritis of the knee: a randomised, double blind, placebo controlled multicentre trial. Hyaluronan Multicentre Trial Group. Ann Rheum Dis 55(7):424-431
100. Bunyaratavej N, Chan KM, Subramanian N (2001) Treatment of painful osteoarthritis of the knee with hyaluronic acid. Results of a multicenter Asian study. J Med Assoc Thai 84(Suppl 2):S576-S581

101. Jubb RW et al (2003) A one-year, randomised, placebo (saline) controlled clinical trial of 500-730 kDa sodium hyaluronate (Hyalgan) on the radiological change in osteoarthritis of the knee. Int J Clin Pract 57(6):467-474

102. Chevalier $X$ et al (2010) Single, intra-articular treatment with $6 \mathrm{~mL}$ hylan G-F 20 in patients with symptomatic primary osteoarthritis of the knee: a randomised, multicentre, double-blind, placebo controlled trial. Ann Rheum Dis 69(1):113-119

103. Petrella RJ, DiSilvestro MD, Hildebrand C (2002) Effects of hyaluronate sodium on pain and physical functioning in osteoarthritis of the knee: a randomized, double-blind, placebo-controlled clinical trial. Arch Intern Med 162(3):292-298

104. Strand V et al (2012) A multicenter, randomized controlled trial comparing a single intra-articular injection of Gel-200, a new cross-linked formulation of hyaluronic acid, to phosphate buffered saline for treatment of osteoarthritis of the knee. Osteoarthr Cartil 20(5):350-356

\section{Submit your manuscript to a SpringerOpen ${ }^{\odot}$ journal and benefit from:}

- Convenient online submission

- Rigorous peer review

- Open access: articles freely available online

- High visibility within the field

- Retaining the copyright to your article

Submit your next manuscript at $\boldsymbol{\nabla}$ springeropen.com 